\title{
Modelo Estocástico para o Algoritmo de Shan de Passo Variável Modificado
}

\author{
José Gil F. Zipf, Orlando J. Tobias e Rui Seara
}

\begin{abstract}
Resumo-Este trabalho discute o algoritmo LMS de passo variável proposto por Shan. Tal algoritmo apresenta (em algumas situações) problemas de convergência que são aqui corrigidos a partir de uma reformulação na expressão de ajuste do passo de adaptação. Além disso, é proposto um modelo para o algoritmo resultante. Simulações numéricas, comparando os resultados obtidos a partir do método de Monte Carlo e do modelo proposto, permitem avaliar tanto o novo algoritmo quanto a qualidade da predição obtida.
\end{abstract}

Palavras-chave-Algoritmos de passo variável, Algoritmo LMS, Filtros adaptativos.

Abstract-This paper discusses the variable step-size LMS algorithm proposed by Shan. Such algorithm presents (in some situations) convergence problems here corrected by reformulating the step-size adjusting expression. In addition, a model for the resulting algorithm is proposed. Numerical simulations, comparing the obtained results from the Monte Carlo method and the proposed model, permit to assess both the new algorithm and the model predictions.

Keywords-Variable step-size adaptive algorithms, LMS algorithm, Adaptive filters.

\section{INTRODUÇÃO}

Desde o seu surgimento, o algoritmo LMS é o mais utilizado em aplicações de filtragem adaptativa devido à sua simplicidade e robustez [1], [2]. No algoritmo LMS, o parâmetro de passo de adaptação, denotado aqui por $\mu$, controla a taxa de convergência do filtro adaptativo e também determina o erro quadrático médio em regime permanente. Como a velocidade de convergência é proporcional ao valor do passo de adaptação, a escolha de um valor elevado de $\mu$ conduz a uma rápida convergência dos coeficientes. Essa escolha, porém, resulta em um aumento do erro em excesso quando comparado ao predito pela solução de Wiener [3].

Em aplicações de identificação de sistemas [2], cancelamento de ruído [2], cancelamento de eco [2], equalização de canal [2], dentre outras, muitas vezes se faz necessário atingir uma elevada taxa de convergência, concomitantemente, com um pequeno desajuste em regime permanente. Nesse contexto, os denominados algoritmos

José Gil F. Zipf e Rui Seara, LINSE-Laboratório de Circuitos e Processamento de Sinais, Depto. de Eng. Elétrica, Universidade Federal de Santa Catarina, Florianópolis, SC, E-mails: \{gil, seara\}@linse.ufsc.br.

Orlando J. Tobias, LINSE/UFSC e Departamento de Engenharia Elétrica e Telecomunicações, Universidade Regional de Blumenau, SC, E-mail: tobias.oj@ieee.org.

Este trabalho foi parcialmente financiado pelo Conselho Nacional de Desenvolvimento Científico e Tecnológico (CNPq).
LMS de passo variável (VSLMS), ajustando dinamicamente o valor do passo de adaptação, podem satisfazer tal exigência.

Várias estratégias para realizar o ajuste do passo de adaptação vêm sendo propostas na literatura. Tais estratégias baseiam-se no gradiente [3]-[10], no erro quadrático [11]-[13], na função de autocorrelação do erro [14], no valor absoluto do erro de adaptação [15], na normalização do vetor de erro [16], no ajuste proporcional aos valores absolutos dos elementos do vetor de coeficientes [17]-[19] e em outros métodos não convencionais [20]-[22].

Os algoritmos baseados no gradiente ajustam o passo de adaptação com base no valor numérico do gradiente do erro quadrático de estimação. Em [4], é apresentado um dos primeiros algoritmos a utilizar essa abordagem, denominado aqui algoritmo de Shan. Tal algoritmo se mostra bastante simples e eficaz, apresentando, porém, um problema elementar de convergência que é quando o gradiente médio resultar em um valor negativo. Assim como em outros algoritmos VSLMS recentemente publicados, não é também considerado em [4] qualquer modelo analítico que descreva o comportamento desse algoritmo, permitindo realizar análises e reflexões quanto ao seu desempenho.

Neste artigo, é revisitado o algoritmo VSLMS proposto em [4], apresentando uma solução eficaz ao problema de convergência desse algoritmo. Também, propõe-se um modelo estocástico para o comportamento médio do vetor de coeficientes e para a curva de aprendizagem do algoritmo adaptativo modificado em questão. A precisão do modelo analítico proposto é verificada através de resultados de simulação.

\section{AlgORITMO DE SHAN}

Considere, então, o problema de identificação de sistemas mostrado na Fig. 1.

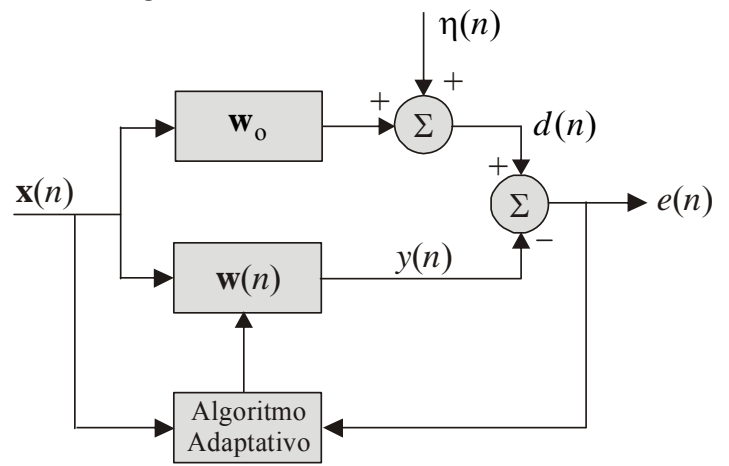

Fig. 1. Diagrama de blocos para identificação de sistemas. 
A saída do sistema a ser identificado (Fig. 1) é dada por

$$
d(n)=\mathbf{w}_{\mathrm{o}}^{\mathrm{T}} \mathbf{x}(n)+\eta(n)
$$

onde $\mathbf{x}(n)=[x(n) x(n-1) \cdots x(n-N+1)]^{\mathrm{T}}$ denota o vetor de entrada, com $\{x(n)\}$ representando um processo de média zero e variância $\sigma_{x}^{2}, \eta(n)$ é um ruído de medição com média zero e variância $\sigma_{\eta}^{2}$, descorrelacionado de qualquer outro sinal no sistema, e $\quad \mathbf{w}_{\mathrm{o}}(n)=\left[w_{\mathrm{o}}(n) w_{\mathrm{o}}(n-1) \cdots\right.$ $\left.w_{\mathrm{o}}(n-N+1)\right]^{\mathrm{T}}$ é o vetor de coeficientes do sistema a ser identificado. Da Fig. 1, o sinal de erro é

$$
e(n)=d(n)-y(n)
$$

onde $y(n)=\mathbf{w}^{\mathrm{T}}(n) \mathbf{x}(n)$ é a saída do filtro adaptativo e $\mathbf{w}(n)=\left[\begin{array}{lll}w(n) & w(n-1) \cdots w(n-N+1)\end{array}\right]^{\mathrm{T}}, \quad$ o $\quad$ vetor de coeficientes do filtro. A equação de atualização do vetor de coeficientes, baseada no algoritmo LMS, é escrita como

$$
\mathbf{w}(n+1)=\mathbf{w}(n)+\mu(n) e(n) \mathbf{x}(n)
$$

onde $\mu(n)$ representa o passo variável de adaptação.

O algoritmo de Shan [4] utiliza uma estimativa da correlação média, entre o erro de adaptação e o sinal de entrada, dada por

$$
\nabla(n)=\lambda \nabla(n-1)+(1-\lambda) e(n) \bar{x}(n)
$$

onde

$$
\bar{x}(n)=\frac{1}{M} \sum_{k=n-M+1}^{n} x(k)
$$

com $\lambda$ caracterizando um fator de esquecimento. $\mathrm{O}$ passo de adaptação é então obtido por

$$
\mu(n)=\alpha \nabla(n)
$$

onde $\alpha$ é uma constante positiva menor do que a unidade. A limitação da variação do passo de adaptação é necessária para garantia da estabilidade do algoritmo, devendo satisfazer a seguinte desigualdade [2]:

$$
0<\mu(n)<\frac{2}{3 \operatorname{tr}[\mathbf{R}]}
$$

onde $\mathbf{R}=E\left[\mathbf{x}(n) \mathbf{x}^{\mathrm{T}}(n)\right]$ é a matriz de autocorrelação do sinal de entrada.

\section{ModificAÇÃo Proposta nO Algoritmo}

O ajuste do passo de adaptação deve se basear na maior ou na menor distância em relação ao ponto ótimo, dado pela solução de Wiener. Observa-se, de (6), que um valor negativo do gradiente médio resulta em um passo de adaptação negativo, sendo limitado a zero pela desigualdade dada em (7). Isso leva o algoritmo LMS a ter um comportamento inadequado. Propõe-se, então, a utilização do valor absoluto do gradiente médio estimado como sendo uma medida mais confiável de distância em relação ao valor do erro quadrático mínimo. Com isso, a seguinte modificação é proposta em (6):

$$
\mu(n)=\alpha|\nabla(n)|
$$

Esta alteração, então, supera os problemas de convergência do algoritmo de Shan [4] anteriormente mencionados como também mantém a simplicidade do algoritmo que é caracterizada pelo seu reduzido aumento de complexidade computacional se comparado ao LMS convencional.

\section{Modelagem}

O modelo do algoritmo de passo variável (VSLMS) aqui analisado é determinado considerando as seguintes hipóteses:

i) O passo variável $\mu(n)$ é assumido independente e descorrelacionado de qualquer outro sinal no sistema.

ii) $E\left[\mu^{2}(n)\right]=E^{2}[\mu(n)]$.

As hipóteses (i) e (ii) são válidas na condição de adaptação lenta do passo variável $\mu(n)$.

\section{A. Comportamento Médio do Vetor de Coeficientes}

Seja a estimativa do vetor gradiente $\mathbf{g}(n)$ dada por

$$
\mathbf{g}(n)=\lambda \mathbf{g}(n-1)+(1-\lambda) e(n) \mathbf{x}(n) .
$$

A estimativa do gradiente médio (ou correlação média entre o erro de adaptação e o sinal de entrada), obtida em (4), pode ser reescrita como

$$
\nabla(n)=\frac{1}{M} \sum_{i=0}^{M-1} g_{i}(n) .
$$

Em (11), $g_{i}(n)$ denota o $i$-ésimo componente do vetor $\mathbf{g}(n)$. Calculando o valor esperado de ambos os lados de (10), tem-se

$$
E[\mathbf{g}(n)]=\lambda E[\mathbf{g}(n-1)]+(1-\lambda)\{\mathbf{p}-\mathbf{R} E[\mathbf{w}(n)]\} .
$$

Então, obtém-se o valor esperado de (11), determinado por

$$
E[\nabla(n)]=\frac{1}{M} \sum_{i=0}^{M-1} E\left[g_{i}(n)\right]
$$

e, assim, o valor esperado do passo de adaptação

$$
E[\mu(n)]=\alpha|E[\nabla(n)]| .
$$

Considerando (i) e as expressões (12)-(14), o valor esperado do vetor de coeficientes do filtro é

$$
E[\mathbf{w}(n+1)]=E[\mathbf{w}(n)]+E[\mu(n)]\{\mathbf{p}-\mathbf{R} E[\mathbf{w}(n)]\}
$$

onde $\mathbf{p}=E[d(n) \mathbf{x}(n)]$ é o vetor de correlação cruzada entre o sinal desejado e o vetor de entrada. 


\section{B. Curva de Aprendizagem}

O modelo para a curva de aprendizagem do algoritmo VSLMS é agora determinado. Seja o vetor de erro nos coeficientes dado por $\mathbf{v}(n)=\mathbf{w}(n)-\mathbf{w}_{\mathrm{o}}$, e a partir de (3), obtém-se a seguinte expressão:

$$
\mathbf{v}(n+1)=\mathbf{v}(n)+\mu(n) e(n) \mathbf{x}(n) .
$$

Representando agora o sinal de erro em função de $\mathbf{v}(n)$, tem-se

$$
\begin{aligned}
e(n) & =d(n)-\left[\mathbf{x}^{\mathrm{T}}(n) \mathbf{w}(n)\right] \\
& =d(n)-\mathbf{x}^{\mathrm{T}}(n) \mathbf{v}(n)-\mathbf{x}^{\mathrm{T}}(n) \mathbf{w}_{\mathrm{o}} .
\end{aligned}
$$

Substituindo (17) em (16), resulta em

$$
\begin{aligned}
\mathbf{v}(n+1)= & \mathbf{v}(n)+[\alpha+\mu(n)][d(n) \mathbf{x}(n) \\
& \left.-\mathbf{x}(n) \mathbf{x}^{\mathrm{T}}(n) \mathbf{v}(n)-\mathbf{x}(n) \mathbf{x}^{\mathrm{T}}(n) \mathbf{w}_{\mathrm{o}}\right] .
\end{aligned}
$$

Definindo então

$$
e_{\mathrm{o}}(n)=d(n)-\mathbf{x}^{\mathrm{T}}(n) \mathbf{w}_{\mathrm{o}}
$$

pode-se escrever

$$
\mathbf{v}(n+1)=\mathbf{v}(n)+(\alpha+\mu(n))\left[e_{\mathrm{o}}(n) \mathbf{x}(n)-\mathbf{x}(n) \mathbf{x}(n)^{\mathrm{T}} \mathbf{v}(n)\right]
$$

determinando assim o seguinte produto de vetores:

$$
\begin{aligned}
\mathbf{v}(n+1) \mathbf{v}^{\mathrm{T}}( & +1)=\mathbf{v}(n) \mathbf{v}^{\mathrm{T}}(n) \\
& +\mu(n) \mathbf{v}(n) \mathbf{v}^{\mathrm{T}}(n) \mathbf{x}(n) \mathbf{x}^{\mathrm{T}}(n) \\
& +\mu(n) e_{\mathrm{o}}(n) \mathbf{v}(n) \mathbf{x}^{\mathrm{T}}(n) \\
& -\mu(n) \mathbf{x}(n) \mathbf{x}^{\mathrm{T}}(n) \mathbf{v}(n) \mathbf{v}^{\mathrm{T}}(n) \\
& +\mu^{2}(n) \mathbf{x}(n) \mathbf{x}^{\mathrm{T}}(n) \mathbf{v}(n) \mathbf{v}^{\mathrm{T}}(n) \mathbf{x}(n) \mathbf{x}^{\mathrm{T}}(n) \\
& -\mu^{2}(n) e_{\mathrm{o}}(n) \mathbf{x}(n) \mathbf{x}^{\mathrm{T}}(n) \mathbf{v}(n) \mathbf{x}^{\mathrm{T}}(n) \\
& +\mu(n) e_{\mathrm{o}}(n) \mathbf{x}(n) \mathbf{v}^{\mathrm{T}}(n) \\
& -\mu^{2}(n) e_{\mathrm{o}}(n) \mathbf{x}(n) \mathbf{v}^{\mathrm{T}}(n) \mathbf{x}(n) \mathbf{x}^{\mathrm{T}}(n) \\
& +\mu^{2}(n) e_{\mathrm{o}}(n) \mathbf{x}(n) \mathbf{x}^{\mathrm{T}}(n) .
\end{aligned}
$$

Agora, arranjando os termos de (21), tem-se

$$
\begin{aligned}
& \mathbf{v}(n+1) \mathbf{v}^{\mathrm{T}}(n+1)=\mathbf{v}(n) \mathbf{v}^{\mathrm{T}}(n) \\
& -\mu(n)\left[\mathbf{x}(n) \mathbf{x}^{\mathrm{T}}(n) \mathbf{v}(n) \mathbf{v}^{\mathrm{T}}(n)+\mathbf{v}(n) \mathbf{v}^{\mathrm{T}}(n) \mathbf{x}(n) \mathbf{x}^{\mathrm{T}}(n)\right] \\
& +\mu^{2}(n)\left[\mathbf{x}(n) \mathbf{x}^{\mathrm{T}}(n) \mathbf{v}(n) \mathbf{v}^{\mathrm{T}}(n) \mathbf{x}(n) \mathbf{x}^{\mathrm{T}}(n)\right] \\
& +\mu^{2}(n) e_{\mathrm{o}}(n)^{2} \mathbf{x}(n) \mathbf{x}^{\mathrm{T}}(n) \\
& +\mu(n) e_{\mathrm{o}}(n)\left\{\left[\mathbf{x}(n) \mathbf{v}^{\mathrm{T}}(n)-\right.\right. \\
& \left.\quad[\alpha+\mu(n)] \mathbf{x}(n) \mathbf{v}^{\mathrm{T}}(n) \mathbf{x}(n) \mathbf{x}^{\mathrm{T}}(n)\right] \\
& \left.+\left[\mathbf{x}(n) \mathbf{v}^{\mathrm{T}}(n)-[\alpha+\mu(n)] \mathbf{x}(n) \mathbf{v}^{\mathrm{T}}(n) \mathbf{x}(n) \mathbf{x}^{\mathrm{T}}(n)\right]^{\mathrm{T}}\right\} .
\end{aligned}
$$

Definindo $\mathbf{K}(n)$ como a matriz de covariância do vetor de erro nos coeficientes, obtida a partir de $\mathbf{K}(n)=E[\mathbf{v}(n)$ $\left.\mathbf{v}^{\mathrm{T}}(n)\right]$, tomando o valor esperado de ambos os lados de (22), e considerando as hipóteses (i) e (ii), obtém-se

$$
\begin{aligned}
\mathbf{K}(n+1)=\mathbf{K}(n)-E[\mu(n)][\mathbf{R K}(n)+\mathbf{K}(n) \mathbf{R}] \\
\quad+E^{2}[\mu(n)][\mathbf{R} \operatorname{tr}[\mathbf{R K}(n)]+2 \mathbf{R K}(n) \mathbf{R}] \\
+E^{2}[\mu(n)] \mathbf{R} \sigma_{\eta}^{2} .
\end{aligned}
$$

Finalmente, a expressão para a curva de aprendizagem é dada por

$$
J(n)=E\left[e^{2}(n)\right]=\sigma_{\eta}^{2}+\operatorname{tr}[\mathbf{R K}(n)]
$$

\section{Resultados de Simulação}

Nesta seção, são apresentadas algumas simulações numéricas visando avaliar o novo algoritmo de passo variável como também verificar a precisão do modelo proposto para o algoritmo. Para tal, considera-se então um problema de identificação de sistemas. A planta utilizada é obtida a partir de uma janela de Hanning com 9 coeficientes dada por $\mathbf{w}_{\mathrm{o}}=\left[\begin{array}{lllllll}0,000 & 0,1170 & 0,4132 & 0,7500 & 0,9698 & 0,7500 & 0,4132\end{array}\right.$ $\left.\begin{array}{lll}0,1170 & 0,000\end{array}\right]^{\mathrm{T}}$. Para o sinal de entrada, são considerados tanto um ruído branco como um sinal colorido, ambos Gaussianos de médias zero e variâncias unitárias $\left(\sigma_{x}^{2}=1\right)$. $\mathrm{O}$ sinal colorido é obtido de um processo AR(1), dado por $x(n)=a x(n-1)+u(n)$, onde $a=0,4$ e $u(n)$ é um ruído branco Gaussiano com variância $\sigma_{x}^{2}=1$. A dispersão dos autovalores da matriz de entrada, para o sinal correlacionado, é $\chi=5$. Também, é introduzido um ruído aditivo de medição com variância $\sigma_{\eta}^{2}=0,01 \quad(\mathrm{SNR}=20 \mathrm{~dB})$.

\section{Exemplo 1: Modificação do Algoritmo de Shan}

Nesta simulação, é utilizado um sinal colorido de entrada (obtido por um processo $\mathrm{AR}(1)$, como apresentado anteriormente). Os resultados de simulação são obtidos através do método de Monte Carlo (MC), considerando uma média de 200 realizações independentes. Na iteração $n=300$, é provocada uma alteração na planta, mudando o sinal de todos os seus coeficientes. Dessa forma, é verificado o comportamento dos algoritmos frente uma perturbação. Da Fig. 2(a), observa-se que o algoritmo original [4] não converge após a referida alteração da planta, visto que o gradiente médio se torna negativo. Na Fig. 2(b), é mostrado o comportamento do algoritmo proposto que inclui a modificação de (6) para (8). O algoritmo proposto agora converge sem quaisquer problemas. 


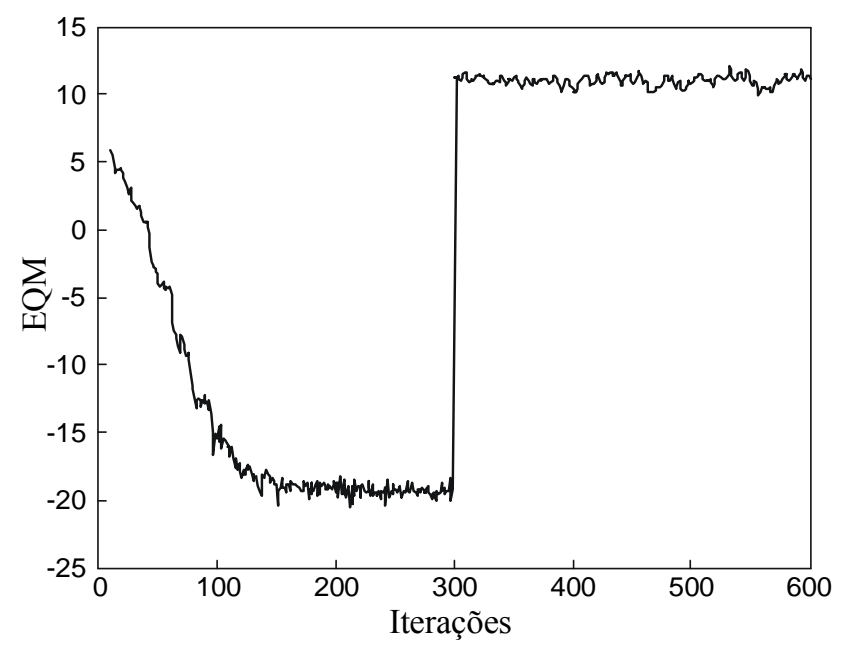

(a)

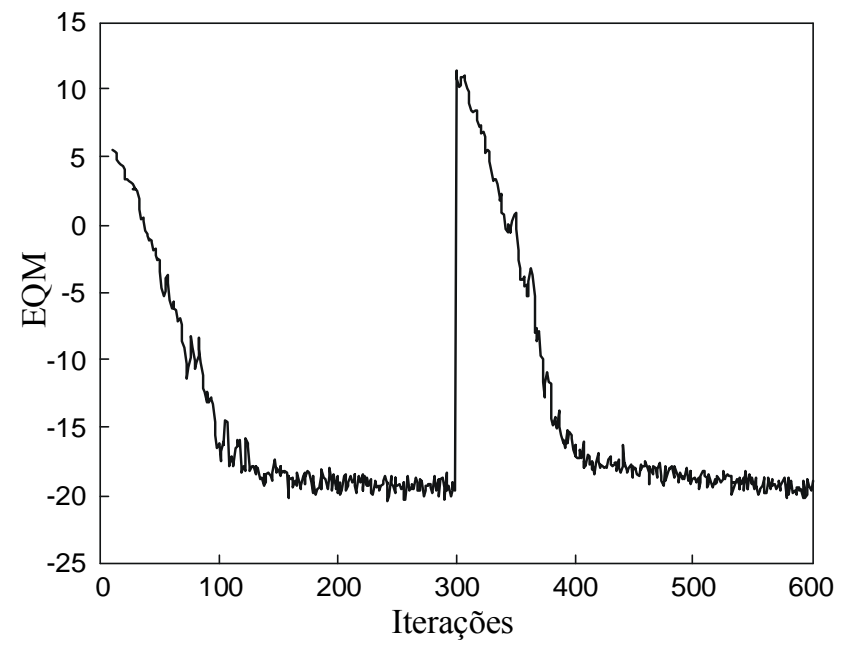

(b)

Fig. 2. Exemplo 1. Evolução do EQM. (a) Algoritmo original. (b) Algoritmo modificado.

\section{Exemplo 2: Resultados do Modelo Proposto}

Neste exemplo, são apresentados os resultados obtidos através de simulação $\mathrm{MC}$ e a partir do modelo proposto, para sinais de entrada ruído branco (Fig. 3) e correlacionado (Fig. 4). Dessas figuras, observa-se um muito bom casamento entre os resultados de simulação $\mathrm{MC}$ e aqueles preditos pelo modelo para a evolução do passo de adaptação [Figs. 3(a) e 4(a) ] como também para o vetor de coeficientes médio [Figs. 3(b) e 4(b)] e erro quadrático médio [Figs. 3(c) e 4(c)].

\section{Exemplo 3: Validação da Hipótese (9)}

Neste exemplo, é verificada através de simulações a hipótese (9) aqui considerada. A Fig. 5 mostra a evolução das seguintes quantidades $E\left[\mu^{2}(n)\right]$ e $E^{2}[\mu(n)]$, obtida através de simulação MC, considerando 200 realizações independentes para sinal de entrada ruído branco. Dessa figura, constata-se um casamento muito bom entre as duas curvas, indicando a razoabilidade da hipótese em questão.

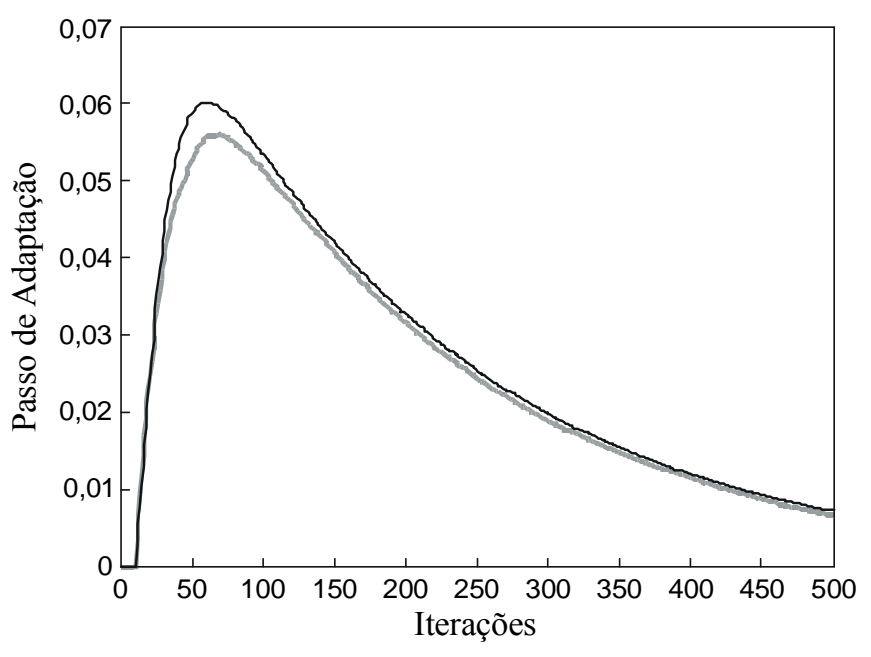

(a)

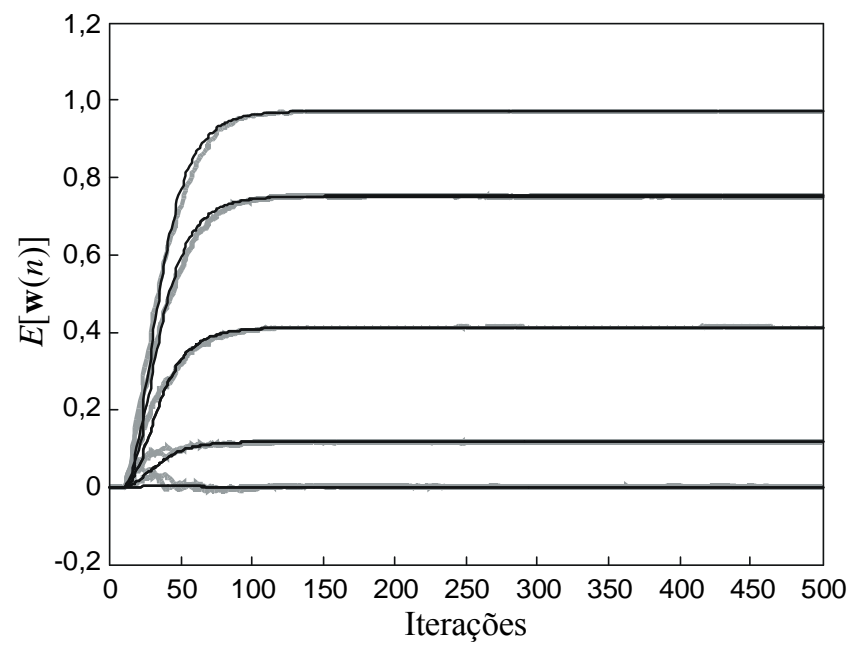

(b)

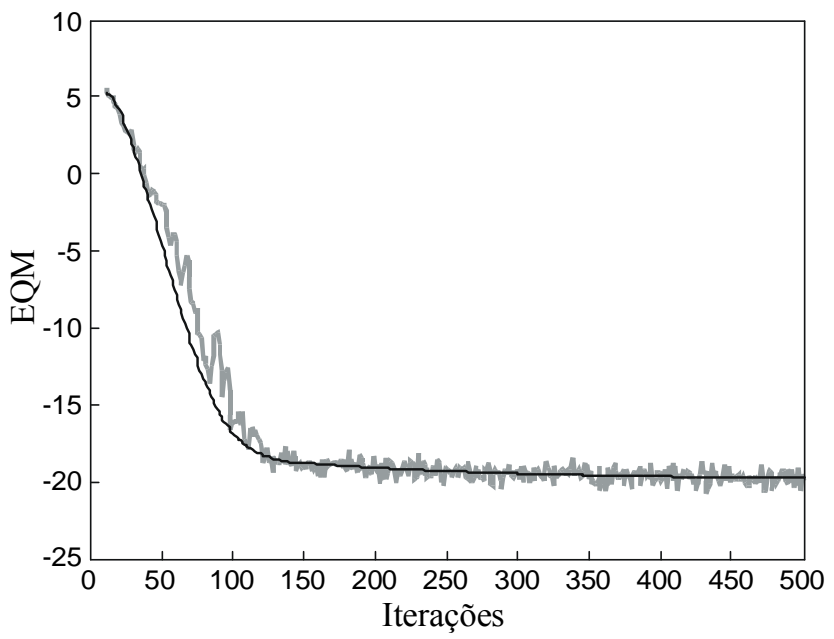

(c)

Fig. 3. Exemplo 2. Sinal de entrada ruído branco. (a) Curvas de ajuste do passo de adaptação. (b) Vetor de coeficientes médio. (c) Curva de aprendizagem. 


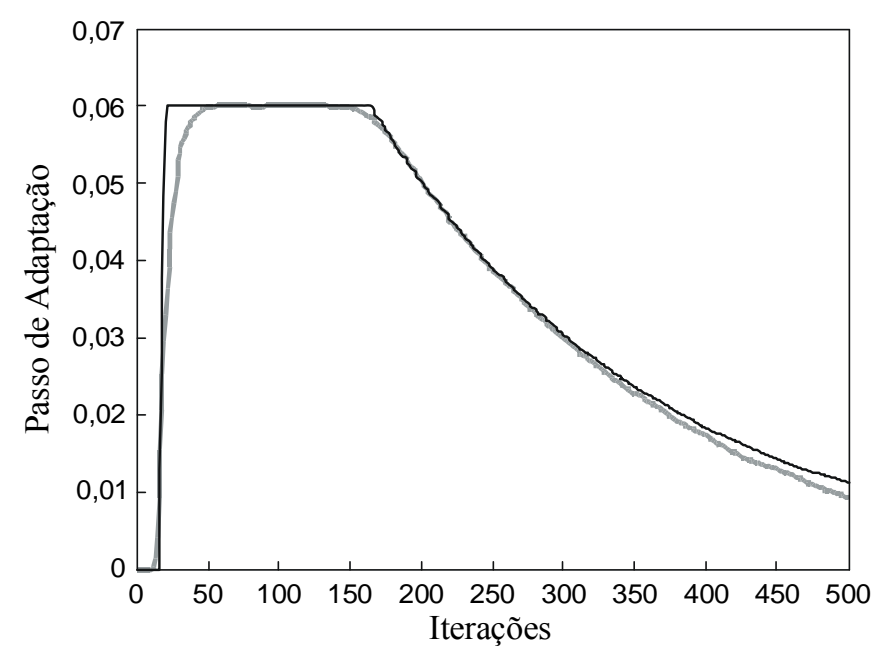

(a)

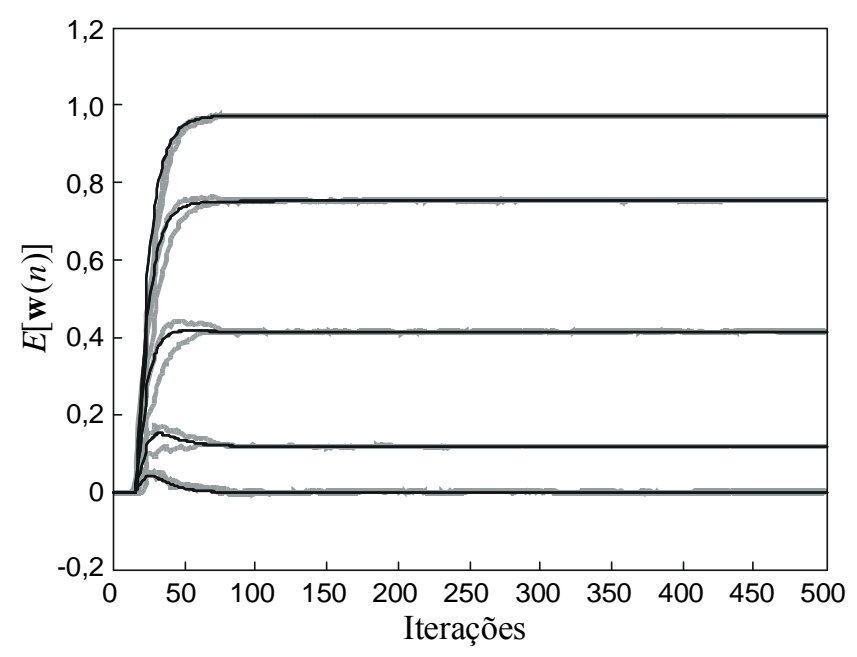

(b)

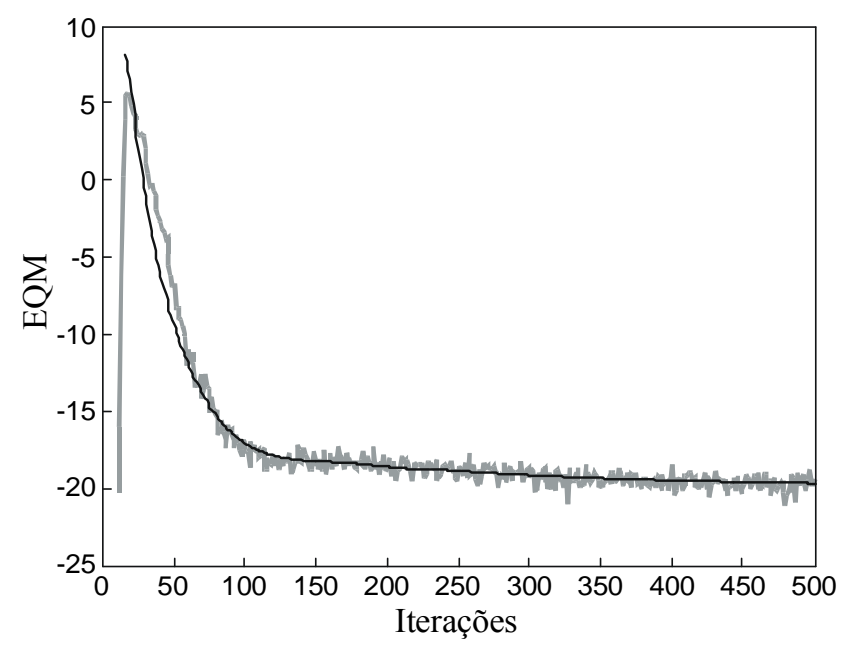

(c)

Fig. 4. Exemplo 2. Sinal de entrada colorido. (a) Curvas de ajuste do passo de adaptação. (b) Vetor de coeficientes médio. (c) Curva de aprendizagem.

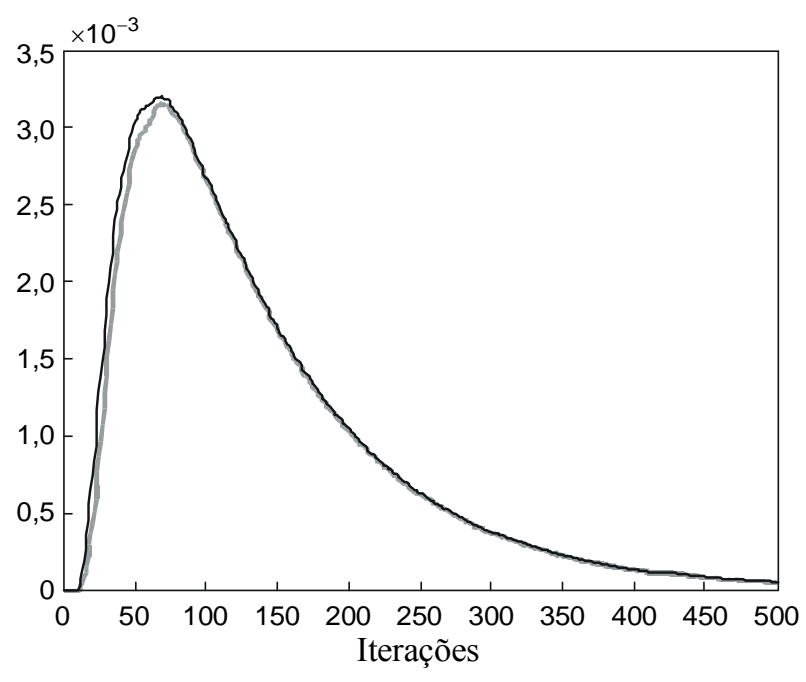

Fig. 5. Evolução de $E\left[\mu^{2}(n)\right]$ e $E^{2}[\mu(n)]$.

\section{CONCLUSÕES}

Neste trabalho, é realizada uma modificação no algoritmo LMS de passo variável proposto por Shan [4]. Tal modificação visou o aprimoramento do referido algoritmo com vistas à sua estabilidade. Com a nova expressão de ajuste do passo de adaptação, fruto da modificação considerada, um modelo para o algoritmo de Shan (modificado) é determinado. A partir de simulações numéricas, é verificado o desempenho do novo algoritmo e a precisão do modelo proposto, quando confrontados com resultados obtidos a partir de simulações Monte Carlo.

\section{REFERÊNCIAS}

[1] B. Widrow and M. Hoff, "Adaptive switching circuits", in Proc. IRE Western Electronic Show and Convention, New York, USA, Part 4, Aug. 1960, pp. 96-104.

[2] S. Haykin, Adaptive Filter Theory, $4^{\text {th }}$ ed., Upper Saddle River, NJ: Prentice Hall, 2002.

[3] R. W. Harris, D. M. Chabries, and F. A. Bishop, "A variable step (VS) adaptive filter," IEEE Trans. Acoust., Speech, Signal Process., vol. ASSP-34, no. 2, pp. 309-316, Apr. 1986.

[4] T. J. Shan and T. Kailath, "Adaptive algorithms with an automatic gain control feature," IEEE Trans. Circuits Syst., vol. CAS-35, no. 1, pp. 122-127, Jan. 1988.

[5] J. C. Richards, M. A. Webster, and J. C. Principe, "A gradient-based variable step-size LMS algorithm," in Proc. IEEE Southeastcon, Williamsburg, USA, vol. 2, Apr. 1991, pp. 1083-1087.

[6] V. J. Mathews and Z. Xie, "A stochastic gradient adaptive filter with gradient adaptive step size," IEEE Trans. Signal Process., vol. 41, no. 6, pp. 2075-2087, June 1993.

[7] A. I. Sulyman and A. Zerguine, "Convergence and steady state analysis of a variable step-size normalized LMS algorithm," Proc. IEEE Int. Symp. Signal Processing and Its Applications (ISSPA), Paris, France, vol. 2, July 2003, pp. 591-594.

[8] B. Farhang-Boroujeny, "Variable step size LMS algorithm - New developments and experiments," IEE Proceedings - Vision, Image, Signal Process., vol. 141, no. 5, pp. 311-317, Oct. 1994.

[9] J. Okello, Y. Itoh, Y. Fukui, I. Nakanishi, and M. Kobayashi, "A new modified variable step size for the LMS algorithm," in Proc. IEEE Int. Symp. Circuits and Systems (ISCAS), Monterey, USA, vol. 5, Jun. 1998, pp. 170-173. 
[10] W. P. Ang and B. Farhang-Boroujeny, "A new class of gradient adaptive step-size LMS algorithms," IEEE Trans. Signal Process., vol. 49, no. 4, pp. 805-810, Apr. 2001.

[11] R. H. Kwong and E. W. Johnston, "A variable step size LMS algorithm," IEEE Trans. Signal Process., vol. 40, no. 7, pp. 1633-1642, July 1992.

[12] I. Nakanishi and Y. Fukui, "A new adaptive convergence factor algorithm with the constant damping parameter," IEICE Trans. Fundamentals, vol. E78-A, no. 6, pp. 649-655, Jun. 1995.

[13] M. H. Costa and J. C. M. Bermudez, "A robust variable step size algorithm for LMS adaptive filters," in Proc. IEEE Int. Conf. Acoust., Speech, Signal Process., Toulouse, France, vol. 3, May 2006, pp. 93-96.

[14] T. Aboulnasr and K. Mayyas, "A robust variable step-size LMS-type algorithm: analysis and simulations", IEEE Trans. Signal Process., vol. 45, no. 3, pp. 631-639, Mar. 1997.

[15] D. W. Kim, J. H. Hoi, Y. S. Choi, C. H. Jeon, and H. Y. Ko, "A VS-LMS algorithm using normalized absolute estimation error", in Proc. IEEE Digital Signal Processing Applications (TENCON), Perth, Australia, vol. 2, Nov. 2006, pp. 692-697.

[16] Z. Ramadan and A. Poularikas, "A robust variable step-size LMS algorithm using error-data normalization," in Proc. IEEE Southeastcon, Huntsville, USA, Apr. 2005, pp. 219-224.

[17] B. Rohani and K. S. Chung, "A modified LMS algorithm with improved convergence," in Proc. IEEE Singapore Int. Conf. Communication Systems, Singapore, Nov. 1994, pp. 845-849.

[18] D. L. Duttweiler, "Proportionate normalized LMS adaptation in echo cancellers", IEEE Trans. Speech Audio Process., vol. 8, no. 5, pp. 508-518, Sept. 2000.

[19] J. Benesty and S. L. Gay, "An improved PNLMS algorithm," in Proc. IEEE Int. Conf. Acoust., Speech, Signal Process., Orlando, USA, May 2002, pp. 1881-1884.

[20] J. Chao, H. Perez, and S. Tsujii, "A fast adaptive filter algorithm using eigenvalue reciprocals as stepsizes," IEEE Trans. Acoust., Speech, Signal Process., vol. 38, no. 8, pp. 1343-1352, Aug. 1990.

[21] M. A. Khasawneh and K. A. Mayyas, "A newly derived variable degree variable step size LMS algorithm", Int. Journal Electronics, vol. 79, no. 3, pp. 255-264, Sept. 1995.

[22] Y. Wei and S. B. Gelfand, "Noise-constrained least mean squares algorithm," IEEE Trans. Signal Process., vol. 49, no. 9, pp. 1961-1970, Sept. 2001. 